\title{
The classification of exceptional Dehn surgeries on 2-bridge knots
}

\author{
Mark BRITtenham AND Ying-QING WU
}

We will classify all exceptional Dehn surgeries on 2-bridge knots according to whether they produce reducible, toroidal, or Seifert fibered manifolds.

\section{Introduction.}

A nontrivial Dehn surgery on a hyperbolic knot $K$ in $S^{3}$ is exceptional if the resulting manifold is either reducible, or toroidal, or a Seifert fibered manifold whose orbifold is a sphere with at most three exceptional fibers, called a small Seifert fibered space. Thus an exceptional Dehn surgery is non-hyperbolic, and using a version of Thurston's orbifold theorem proved by Boileau and Porti [BP], it can be shown that a non-exceptional surgery on a 2-bridge knot is also hyperbolic, see Remark 6.3. There has been much research into determining how many exceptional surgeries a knot can admit. See the survey articles [Go, Lu] for details.

The purpose of this paper is to classify all exceptional Dehn surgeries on 2-bridge knots. Hatcher and Thurston [HT] have shown that there is no reducible surgery on hyperbolic 2-bridge knots. We will determine all toroidal surgeries and small Seifert fibered surgeries on these knots, which will then complete the classification.

We use $\left[b_{1}, \ldots, b_{n}\right]$ to denote the partial fraction decomposition $1 /\left(b_{1}-\right.$ $\left.1 /\left(b_{2}-\ldots-1 / b_{n}\right) \ldots\right)$. Recall that a 2 -bridge knot $K$ is non-hyperbolic if and only if $K=K_{1 / q}$ for some $q$, in which case $K$ is a $(2, q)$ torus knot, and surgery on $K$ is well understood. $K$ is a twist knot if it is equivalent to some $K_{p / q}$ with $p / q=[b, \pm 2]$ for some integer $b$. Since $[b, \pm 2]=[b \mp 1, \mp 2]$, we may assume that $b$ is even. Let $K(\gamma)$ be the manifold obtained by $\gamma$ surgery on $K$. We always assume that $\gamma \neq \infty$, that is, the surgery is nontrivial. With respect to the standard meridian-longitude pair on $\partial N(K)$, each slope $\gamma$ is identified with a rational number, see Rolfsen's book $[R]$. The following is the main theorem of this paper. Note that part (4) of the theorem is the case of surgery on the Figure 8 knot, and is due to Thurston [Th]. It is included in the theorem for the sake of completeness. 
Theorem 1.1. Let $K$ be a hyperbolic 2-bridge knot.

(1) If $K \neq K_{\left[b_{1}, b_{2}\right]}$ for any $b_{1}, b_{2}$, then $K$ admits no exceptional surgery.

(2) If $K=K_{\left[b_{1}, b_{2}\right]}$ with $\left|b_{1}\right|,\left|b_{2}\right|>2$, then $K(\gamma)$ is exceptional for exactly one $\gamma$, which yields a toroidal manifold. When both $b_{1}$ and $b_{2}$ are even, $\gamma=0$. If $b_{1}$ is odd and $b_{2}$ is even, $\gamma=2 b_{2}$.

(3) If $K=K_{[2 n, \pm 2]}$ and $|n|>1, K(\gamma)$ is exceptional for exactly five $\gamma: K(\gamma)$ is toroidal for $\gamma=0, \mp 4$, and is small Seifert fibered for $\gamma=\mp 1, \mp 2, \mp 3$.

(4) If $K=K_{[2,-2]}$ is the Figure $8 \mathrm{knot}, K(\gamma)$ is exceptional for only nine $\gamma: K(\gamma)$ is toroidal for $\gamma=0,4,-4$, and is Seifert fibered for $\gamma=-1,-2,-3,1,2,3$.

We will use a result of Hatcher and Thurston [HT] to determine all toroidal surgeries on a hyperbolic 2-bridge knot $K$, see Lemma 2.2 below. In general it is more difficult to determine small Seifert fibered surgeries, due to the fact that there is no essential surfaces in such a manifold, unless its Euler number is 0 . Here we will use essential lamination theory developed by Gabai and Oertel [GO]. The readers are referred to [GO] for definitions and basic properties concerning essential branched surfaces and essential laminations, which play a central role in the proof of the theorem. We will use Brittenham's criterion [Br], which says that if $M$ is a small Seifert fibered space containing an essential branched surface $\mathcal{F}$, then each component of $M-\operatorname{Int} N(\mathcal{F})$ is an $I$-bundle over some compact surface $G$. The idea of the proof is to construct essential laminations in surgered manifolds whose complementary regions are not $I$-bundles. We will apply some techniques developed by Delman [De1, De2] and Roberts [Ro] to construct essential laminations in the surgered manifolds.

Acknowledgment. We are grateful to Alan Reid for some very useful comments to an earlier version of this paper. We also thank Steve Bleiler for pointing out that the Orbifold Theorem and the Snappea computer program, two tools used in an earlier version of the paper, are not considered fully proved by many people. The present version has avoided using them in the proof. 


\section{Reducible, toroidal, or Seifert fibered surgeries.}

Reducible surgeries and toroidal surgeries on 2-bridge knots are completely determined by Lemmas 2.1 and 2.2. Certain surgeries on twist knots are shown to be Seifert fibered in Corollary 2.4.

Lemma 2.1 (Hatcher-Thurston). Let $K$ be a 2-bridge knot. Then $K(r)$ is reducible if and only if $K$ is a $(2, q)$ torus knot, and $r=2 q$.

Proof. See [HT, Theorem 2].

Lemma 2.2. Let $K$ be a hyperbolic 2-bridge knot.

(1) If $K(\gamma)$ is toroidal for some $\gamma$, then $K=K_{\left[b_{1}, b_{2}\right]}$ for some $b_{1}, b_{2}$.

(2) If $\left|b_{i}\right|>2$ for $i=1,2$, there is exactly one such $\gamma$. When both $b_{i}$ are even, $\gamma=0$. When $b_{1}$ is odd and $b_{2}$ is even, $\gamma=2 b_{2}$.

(3) If $K=K_{[2 n, 2]}$ and $|n|>1, K(\gamma)$ is toroidal if and only if $\gamma=0$ or -4 . For $K=K_{[2 n,-2]}, \gamma=0$ or 4 .

(4) If $K=K_{[2,-2]}$, then $K(\gamma)$ is toroidal if and only if $\gamma=0$, 4 , or -4 .

Proof. We refer the reader to [HT] for notations. If $K(\gamma)$ is toroidal, there is an essential punctured torus $T$ in the knot exterior. By Theorem 1 of [HT], $T$ is carried by some $\Sigma\left[b_{1}, \ldots, b_{k}\right]$, where $\left[b_{1}, \ldots, b_{k}\right]$ is an expansion of $p / q$. By the proof of Theorem 2 of [HT], we have $0=2-2 g=n(2-k)$, where $g$ is the genus of $T$, and $n$ is the intersection number between $\partial T$ and a meridian of $K$. Therefore $k=2$. This proves (1). The rest follows by determining all the possible expansions of type $\left[b_{1}, b_{2}\right]$ for $p / q$. The boundary slopes of the surfaces can be calculated using Proposition 2 of $[\mathrm{HT}]$. By the proof of [Pr, Corollary 2.1], an incompressible punctured torus $T$ in the exterior of a 2-bridge knot will become an essential torus after surgery along the slope of $\partial T$.

Lemma 2.3. Let $L=k_{1} \cup k_{2}$ be the Whitehead link, which is the 2-bridge link associated to the rational number $p^{\prime} / q^{\prime}=[2,2,-2]$. Let $L\left(\gamma_{1}, \gamma_{2}\right)$ be the manifold obtained by $\gamma_{i}$ surgery on $k_{i}$. If $\gamma_{1}=-1 / n$ and $\gamma_{2}=-1,-2$ or -3 , then $L\left(\gamma_{1}, \gamma_{2}\right)$ is a small Seifert fibered space. 


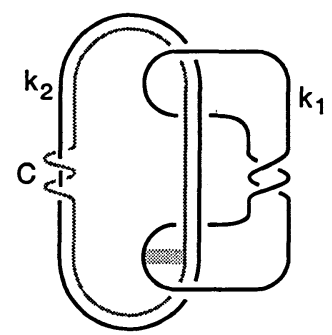

(a)

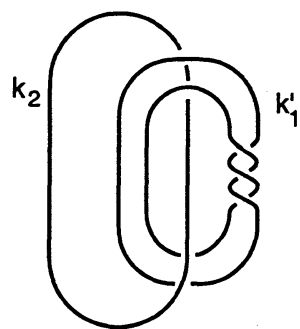

(b)

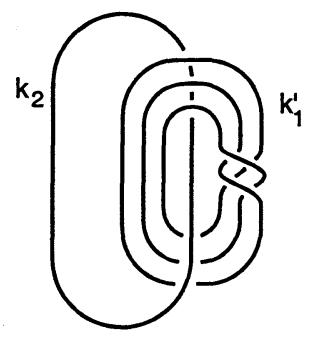

(c)

Figure 1.

Proof. By definition $L\left(\infty, \gamma_{2}\right)$ is the manifold obtained from $S^{3}$ by $\gamma_{2}$ surgery on $k_{2}$. After -1 surgery on $k_{2}$, the knot $k_{1}$ becomes a trefoil knot in $L(\infty,-1)=S^{3}$. Since the exterior of a torus knot is a Seifert fibered space with orbifold a disk with two cones, it is easy to see that all surgeries but one yield Seifert fibered spaces, each having an orbifold a disk with at most three cone points. For this trefoil, the exceptional surgery has coefficient -6 , yielding a reducible manifold. Thus $L(-1 / n,-1)$ is a small Seifert fibered space for any $n$.

After -2 surgery on $k_{2}$, the knot $k_{1}$ becomes a knot in $\mathbb{R} P^{3}=L(\infty,-2)$. The link $L$ is drawn in Figure 1(a), where the curve $C$ is a curve on $\partial N\left(k_{2}\right)$ of slope -2 , so it bounds a disk in $L(\infty,-2)$. Thus a band sum of $k_{1}$ and $C$ forms a knot $k_{1}^{\prime}$ isotopic to $k_{1}$ in $L(\infty,-2)$. The link $L^{\prime}=k_{1}^{\prime} \cup k_{2}$ is shown in Figure 1(b). Using Kirby Calculus one can show that $L(-1 / n,-2)=$ $L^{\prime}(-2-1 / n,-2)$. The exterior of $k_{1}^{\prime}$ in $S^{3}$ is a Seifert fibered space with orbifold a disk with two cones, in which $k_{2}$ is a singular fiber of index 3 . Thus after -2 surgery on $k_{2}$, the manifold $L(\infty,-2)-\operatorname{Int} N\left(k_{1}^{\prime}\right)$ is still Seifert fibered, with orbifold a disk with two cones. The fiber slope on $\partial N\left(k_{1}^{\prime}\right)$ is 6. It follows that all but the 6 surgery on $k_{1}^{\prime}$ in $L(\infty,-2)$ yield small Seifert fibered manifolds. In particular, $L(-1 / n,-2)=L^{\prime}(-2-1 / n,-2)$ are small Seifert fibered manifolds for all $n$.

The proof for $\gamma_{2}=-3$ is similar. One can show that the band sum of $k_{1}$ and the curve $C$ of slope -3 on $\partial N\left(k_{2}\right)$ is isotopic to the curve $k_{1}^{\prime}$ shown in Figure 1(c), which is a $(3,-2)$ torus knot. By the same argument as above one can show that $L(-1 / n,-3)=L(-3-1 / n,-3)$ are small Seifert fibered manifolds for all $n$. 


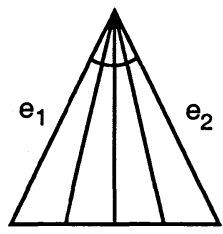

(a)

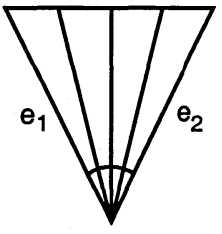

(b)

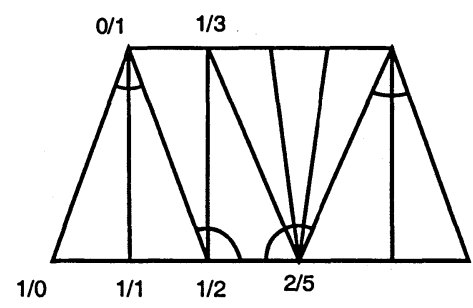

(c)

Figure 2.

Recall that a 2-bridge knot $K$ is a twist knot if $K=K_{p / q}$, and $p / q=$ $[2 n, \pm 2]$ for some $n$.

Corollary 2.4. If $K=K_{p / q}$ is a twist knot with $p / q=[2 n, \pm 2]$, then $K(\gamma)$ is a small Seifert fibered space for $\gamma=\mp 1, \mp 2$ and $\mp 3$.

Proof. Consider the case $p / q=[2 n, 2]$. The proof for $p / q=[2 n,-2]$ is similar. Let $L=k_{1} \cup k_{2}$ be a 2 -bridge link associated to the rational number $p^{\prime} / q^{\prime}=[2,2,-2]$. Notice that after $-1 / n$ surgery on $k_{1}$, the knot $k_{2}$ becomes the knot $K=K_{[2 n, 2]}$ in $S^{3}=L(-1 / n, \infty)$. Therefore by Lemma $6, K(\gamma)=$ $L(-1 / n, \gamma)$ are small Seifert fibered spaces for $\gamma=-1,-2$ and -3 .

\section{Delman's construction, and the proof of Theorem 1.1(1).}

For each rational number $p / q$, there is associated a diagram $D(p / q)$, which is the minimal subdiagram of the Hatcher-Thurston diagram [HT, Figure 4] that contains all minimal paths from $1 / 0$ to $p / q$. See [HT, Figure 5] and [De1]. $D(p / q)$ can be constructed as follows. Let $p / q=\left[a_{1}, \ldots, a_{k}\right]$ be a continued fraction expansion of $p / q$. To each $a_{i}$ is associated a "fan" $F_{a_{i}}$ consisting of $a_{i}$ simplices, see Figure 2(a) and 2(b) for the fans $F_{4}$ and $F_{-4}$. The edges labeled $e_{1}$ are called initial edges, and the ones labeled $e_{2}$ are called terminal edges. The diagram $D(p / q)$ can be constructed by gluing the $F_{a_{i}}$ together in such a way that the terminal edge of $F_{a_{i}}$ is glued to the initial edge of $F_{a_{i+1}}$. Moreover, if $a_{i} a_{i+1}<0$ then $F_{a_{i}}$ and $F_{a_{i+1}}$ have one edge in common, and if $a_{i} a_{i+1}>0$ then they have a 2 -simplex in common. See Figure 2(c) for the diagram of $[2,-2,-4,2]$. Notice that the fans $F_{-2}$ and $F_{-4}$ in the figure share a common triangle. 

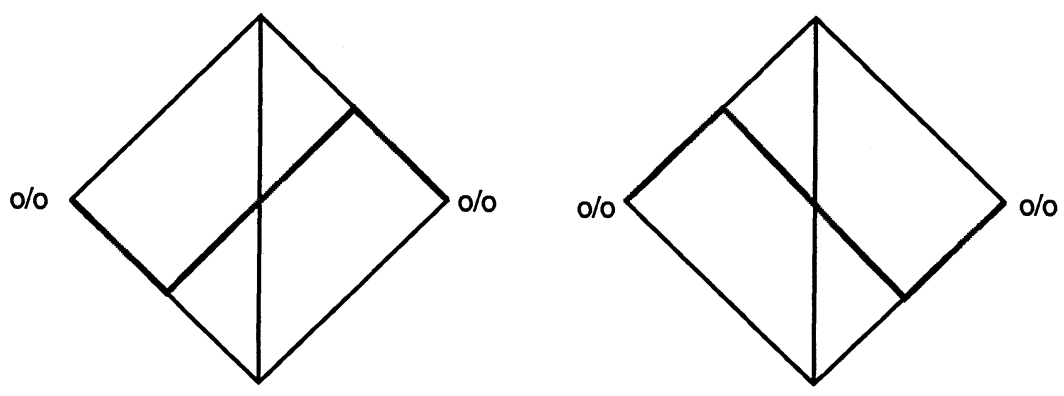

Figure 3.

To each vertex $v_{i}$ of $D(p / q)$ is associated a rational number $r_{i} / s_{i}$. It has one of the three possible parities: odd/odd, odd/even, or even/odd, denoted by $o / o, o / e$, and $e / o$, respectively. Note that the three vertices of any simplex in $D(p / q)$ have mutually different parities.

We consider $D(p / q)$ as a graph on a disk $D$, with all vertices on $\partial D$, containing $\partial D$ as a subgraph. The boundary of $D$ forms two paths from the vertex $1 / 0$ to the vertex $p / q$. The one containing the vertex $0 / 1$ is called the top path, and the one containing the vertex $1 / 1$ is called the bottom path. Edges on the top path are called top edges. Similarly for bottom edges.

Let $\Delta_{1}, \Delta_{2}$ be two simplices in $D(p / q)$ with an edge in common. Assume that the two vertices which are not on the common edge are of parity $o / o$. Then the arcs indicated in Figure 3(a) and (b) are called channels. A path $\alpha$ in $D(p / q)$ is a union of arcs, each of which is either an edge of $D(p / q)$ or a channel.

Let $v$ be a vertex on a path $\alpha$ in $D(p / q)$. Let $e_{1}, e_{2}$ be the edges of $\alpha$ incident to $v$. Then the corner number of $v$ in $\alpha$, denoted by $c(v ; \alpha)$ or simply $c(v)$, is defined as the number of simplices in $D(p / q)$ between the edges $e_{1}$ and $e_{2}$. A path $\alpha$ from $1 / 0$ to $p / q$ is an allowable path if it has at least one channel, and $c(v) \geq 2$ for all $v$ in $\alpha$.

Now assume that $K=K_{p / q}$ is a 2-bridge knot. Then $q$ is an odd number. Recall that $K_{p / q}=K_{p^{\prime} / q}$ if $p^{\prime} \equiv p^{ \pm 1} \bmod q$, and $K_{-p / q}$ is the mirror image of $K_{p / q}$. We may assume without loss of generality that $p$ is even, and $1<p<q$. This is because $K_{(q-p) / q}$ is equivalent to the mirror image of $K_{p / q}$, so the result of $\gamma$ surgery on the first is the same as that of $-\gamma$ surgery on the second. Note that $q-p$ and $p$ have different parity, since $q$ is odd. The following result is due to Delman. See [De1] and [De2, Proposition 3.1]. 
Theorem 3.1 (Delman). Given an allowable path $\alpha$ of $D(p / q)$, there is an essential branched surface $\mathcal{F}$ in $S^{3}-K$ which remains essential after all nontrivial surgeries on the knot $K$.

Lemma 3.2. If there is an allowable path $\alpha$ in $D(p / q)$ such that $c(v)>2$ for some vertex $v$ in $\alpha$, then $K(\gamma)$ is not a small Seifert fibered space for any $\gamma$.

Proof. It was shown in [Br, Corollary 4] that if $\mathcal{F}$ is an essential branched surface in a small Seifert fibered space $M$, then each component of $M-$ Int $N(\mathcal{F})$ is an $I$-bundle over a compact surface $G$, such that the vertical surface $\partial_{v} N(\mathcal{F})$ (also called cusps) is the $I$-bundle over $\partial G$. It has been shown in [De1] that for each vertex $v$ of $\alpha$ there is a component $W_{v}$ of $S^{3}-\operatorname{Int} N(\mathcal{F})$ such that $W_{v}$ is a solid torus whose meridian disk intersects the cusps $c(v)$ times. In particular, if $c(v)>2$ then $W_{v}$ is not an $I$ bundle as above. Since $\mathcal{F}$ is an essential branched surface in $K(\gamma)$, it follows that $K(\gamma)$ is not a small Seifert fibered space.

Lemma 3.3. Suppose $p$ is even, $q$ is odd, and $1<p<q-1$. If $p / q$ does not have partial fraction decomposition of type $\left[r_{1}, r_{2}\right]$, then $D(p / q)$ has an allowable path $\alpha$ such that some vertex $v$ on $\alpha$ has $c(v)>2$.

Proof. Let $\left[a_{1}, \ldots, a_{n}\right]$ be the partial fraction decomposition of $p / q$ such that all $a_{i}$ are even. Then $a_{1} \geq 2$. If $a_{i}=2$ for all $i$, then $p / q=(q-1) / q$, contradicting our assumption. Thus either some $a_{i}<0$, or some $a_{i}>4$. We separate the two cases.

CASE 1. Some $a_{i}<0$.

Let $a_{i}$ be the first negative number. Then $a_{i-1}>0$, so there is a sign change. By [De2] there is a channel $\alpha_{0}$ in $F_{a_{i-1}} \cup F_{a_{i}}$ starting at a bottom edge and ending at a top edge, where $F_{a_{i}}$ is the fan in $D(p / q)$ corresponding to $a_{i}$. Let $\alpha_{1}$ be the part of the bottom path of $D(p / q)$ from the vertex $1 / 0$ to the initial point of $\alpha_{0}$, and let $\alpha_{2}$ be the part of the top path from the end point of $\alpha_{0}$ to the vertex $p / q$. Then $\alpha=\alpha_{1} \cup \alpha_{0} \cup \alpha_{2}$ is an allowable path in $D(p / q)$. We need to show that if $c(v)=2$ for all vertices $v$ on this path, then $p / q=\left[r_{1}, r_{2}\right]$ for some $r_{1}, r_{2}$.

Consider the vertices on $\alpha_{1}$. Since $c\left(v_{i}\right)=2$ for all $v_{i}$, each vertex $v_{i}$ is incident to exactly one non boundary edge $e_{i}$ of $D(p / q)$, which must have the other end on a vertex $v_{i}^{\prime}$ in the top path. If some of these $v_{i}^{\prime}$ are different, 


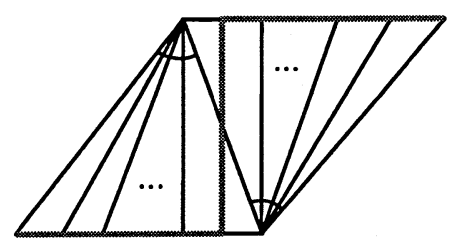

(a)

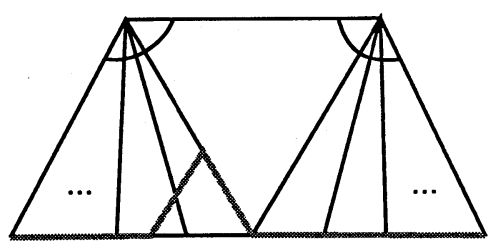

(b)

Figure 4.

then since all faces of $D(p / q)$ are triangles, it is clear that some $v_{j}$ on $\alpha_{1}$ would have at least two non boundary edges, which would be a contradiction. Similarly, each vertex on $\alpha_{2}$ has a unique non boundary edge, leading to a common vertex on the bottom path, so the diagram $D(p / q)$ looks exactly as in Figure 4(a). It is the union of two fans $F_{r_{1}}$ and $F_{r_{2}}$ with $r_{1}>0$, and $r_{2}<0$. Therefore, $p / q=\left[r_{1}, r_{2}\right]$.

\section{CASE 2. Some $a_{i} \geq 4$.}

In this case there is a channel $\alpha_{0}$ with both ends on the bottom path. Construct an allowable path $\alpha=\alpha_{1} \cup \alpha_{0} \cup \alpha_{2}$ with $\alpha_{1}, \alpha_{2}$ in the bottom path. Similar to Case 1 , it can be shown that each vertex on $\alpha_{i}$ has a unique non boundary edge leading to a common vertex $v_{i}^{\prime}$ on the top path, so $D(p / q)$ looks like that in Figure 4(b). In this case $p / q=\left[r_{1}, r_{2}\right]$, with both $r_{i}>0$.

Corollary 3.4. Let $K$ be a 2-bridge knot. If $K \neq K_{\left[b_{1}, b_{2}\right]}$ for any $b_{1}, b_{2}$, then $K(\gamma)$ is non-exceptional for all $\gamma$.

Proof. $K$ is not a $(2, q)$ torus knot, otherwise $K=K_{[2 q \pm 1]}=K_{[2 q, \mp 1]}$. Hence by Lemmas 2.1 and $2.2, K(\gamma)$ is irreducible and atoroidal. By Lemmas 3.3 and $3.2, K(\gamma)$ is not a small Seifert fiber space. Therefore, $K(\gamma)$ is non-exceptional. 


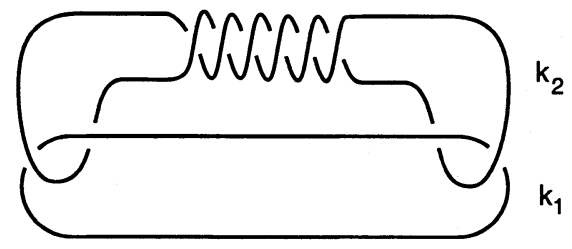

Figure 5.

\section{Surgery on twisted Whitehead links, proof of Theorem 1.1(2).}

A twisted Whitehead link is a two bridge link $L$ associated to a rational number $[2, r,-2]$ for some $r \neq 0$. See Figure 5 for a twisted Whitehead link with $r=-6$. When $r= \pm 2, L$ is a Whitehead link. It has been determined exactly which 2-bridge link complements contain persistent laminations [Wu]. The next lemma shows that if $|r|>2$ then there is a persistent lamination with some desired property.

Recall that a slope $\gamma$ of a knot $K$ is an integral slope if it intersects the meridian of $K$ exactly once.

Lemma 4.1. Let $L=k_{1} \cup k_{2}$ be a twisted Whitehead link associated to the rational number $p / q=[2, r,-2]$. Let $L\left(\gamma_{1}, \gamma_{2}\right)$ be the manifold obtained by $\gamma_{i}$ surgery on $k_{i}$. If $|r|>2$, and one of the $\gamma_{i}$ is not an integral slope, then $L\left(\gamma_{1}, \gamma_{2}\right)$ is not a small Seifert fiber space.

Proof. By considering the mirror image of $L$ if necessary we may assume that $r<0$. If $r$ is even, then $[2, r,-2]$ is a partial fraction decomposition with even coefficients, and $r \leq-4$. There is an allowable path in $D(p / q)$ with two channels, as shown in Figure 6(a), where $r=-4$. If $r$ is odd, then $p / q=[2, r+1,2]$, in which case $D(p / q)$ also has an allowable path with two channels. See Figure 6(b) for the case $r=-3$.

Let $\mathcal{F}$ be the essential branched surface in the link exterior associated to the above allowable path in $D(p / q)$, as constructed in [De2]. There is one solid torus component $V_{i}$ in $S^{3}-\operatorname{Int} N(\mathcal{F})$ for each $k_{i}$, containing $k_{i}$ as a central curve. From the construction of $\mathcal{F}$ one can see that each channel contributes two cusps, one on each $\partial V_{i}$. Actually from [De2, Figure 3.5] we see that the two cusps corresponding to a channel are around two points of $L$ on a level sphere with same orientation. Since each $k_{i}$ intersects the 


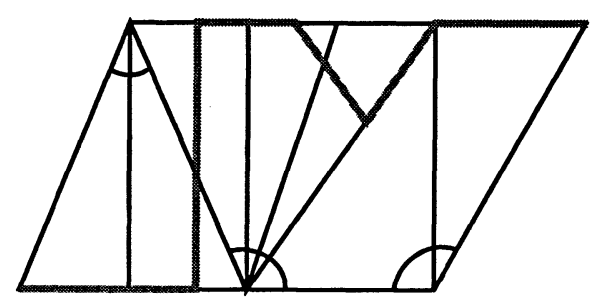

(a)

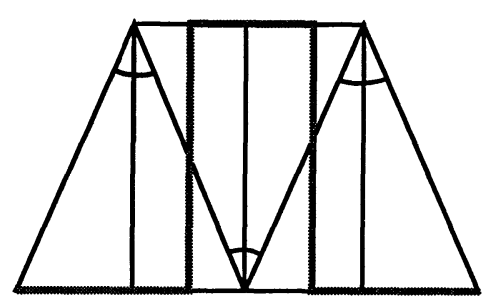

(b)

Figure 6.

sphere at two points with different orientations, those two cusps must be around different components of $L$. One is referred to [Wu] for more details about surgery on 2-bridge links.

As the allowable path above has two channels, each $V_{i}$ has two meridional cusps. Thus $\mathcal{F}$ remains an essential branched surface after surgery on $L$. Moreover, since one of the $\gamma_{i}$ is non-integral, after surgery $V_{i}$ becomes a solid torus whose meridional disk intersects the cusps at least four times. $\mathrm{By}$ [ $\mathrm{Br}$, Corollary 4], the surgered manifold is not a small Seifert fiber space.

Corollary 4.2. Let $K=K_{\left[b_{1}, b_{2}\right]}$ be a two bridge knot with $\left|b_{i}\right|>2$ for $i=1,2$. Then $K(\gamma)$ is exceptional for only one $\gamma$, which yields toroidal manifold. When both $b_{1}$ and $b_{2}$ are even, $\gamma=0$. If $b_{1}$ is odd and $b_{2}$ is even, $\gamma=2 b_{2}$.

Proof. By Lemmas 2.1 and 2.2, $K(\gamma)$ is irreducible, and it is toroidal for exactly one $\gamma$ as described in the corollary. So it remains to show that $K(\gamma)$ is never a small Seifert fiber space.

Since $K$ is a knot, at least one of the $b_{i}$ is an even number. We may assume without loss of generality that $b_{1}=2 n$ for some integer $n$, because $K_{\left[b_{1}, b_{2}\right]}$ is equivalent to $K_{\left[b_{2}, b_{1}\right]}$, by turning the standard diagram for the first knot upside down.

Let $L=k_{1} \cup k_{2}$ be a 2-bridge link associated to the rational number $p / q=\left[2, b_{2},-2\right]$. Notice that after $-1 / n$ surgery on $k_{1}$, the other component $k_{2}$ becomes the knot $K=K_{\left[2 n, b_{2}\right]}$. Therefore, doing $\gamma$ surgery on $K$ is the same as doing $-1 / n$ surgery on $k_{1}$, then doing some $\gamma^{\prime}$ surgery on $k_{2}$. Since 

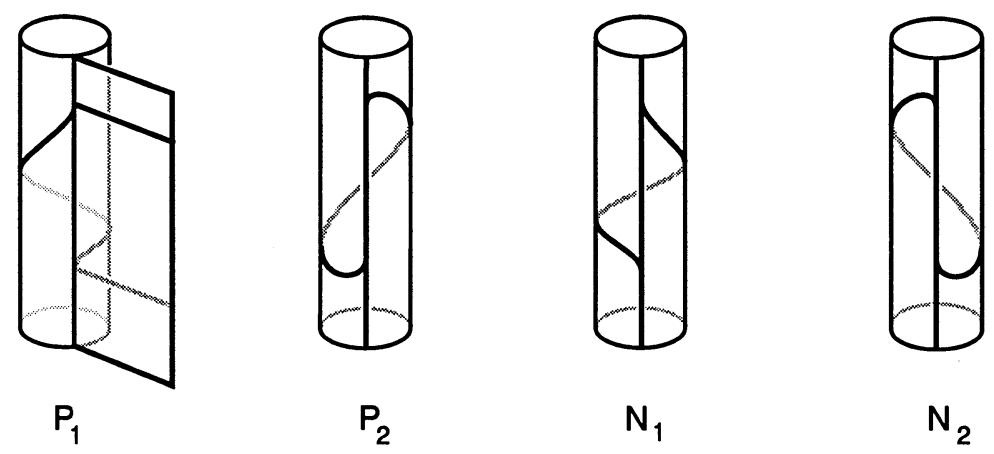

Figure 7.

$-1 / n$ is non integral, and $\left|b_{2}\right|>2$, the result follows from Lemma 4.1.

Corollary 4.3. Let $K=K_{[b, \pm 2]}$ with $|b|>2$. If $\gamma$ is a non integral slope, then $K(\gamma)$ is not a small Seifert fiber space.

Proof. As above, $K(\gamma)=L(\gamma, \pm 1)$, where $L=L_{[2, b,-2]}$. Since $\gamma$ is non integral, the result follows from Lemma 4.1. The result also follows from $[\mathrm{Br}]$.

\section{Roberts' construction of essential branched surfaces.}

In [Ro] Roberts constructed branched surfaces in certain knot complements, which can be extended to essential branched surfaces in $K(\gamma)$ for all $\gamma$ in an infinite interval. We will describe her results and construction in this section, and apply them to surgery on twist knots in the next section.

Let $E(K)=S^{3}-\operatorname{Int} N(K)$ be the exterior of a knot $K$ in $S^{3}$, let $R^{\prime}$ be a (possibly non orientable) compact surface in $S^{3}$ with $\partial R^{\prime}=K$. Let $R=R^{\prime} \cap E(K)$.

Let $S$ be a surface in $E(K)$ which has interior disjoint from $R$, and has a single boundary curve $\partial S=a_{1} \cup b_{1} \cup \ldots \cup a_{n} \cup b_{n}$, where $b_{i}$ are mutually disjoint $\operatorname{arcs}$ on $R$, and $a_{i}$ are $\operatorname{arcs}$ on $T=\partial N(K)$. By specifying a cusp at each $b_{i}$, the union of $R$ and $S$ becomes a branched surface $B=\langle R, S\rangle$ in $E(K)$. The cusps will be assigned in such a way that each $a_{i}$ on $T$ is one of the four types indicated in Figure 7. Note that $\partial B$ is a train track on $T$, and each component of $T-\partial B$ is a digon, i.e a disk with two cusps. 
Remark. The pictures on Figure 7 are mirror images of that in [Ro, Figure 22]. Thus for example, type $P_{1}$ here is of type $N_{1}$ in [Ro]. Apparently we are using different coordinate systems. This paper adopts the convention that the meridian-longitude pair $(m, l)$ on $T$ is chosen so that when $K$ is endowed with the same orientation as that of $l$, the linking number $l k(m, K)=1$, measured using the right hand rule. See $[R]$. With this convention, types $P_{1}, P_{2}$ in Figure 7 will have positive contributions to any slope $\gamma$ carried in the train track.

Let $N(B)$ be a regular neighborhood of the branched surface $B$ in $E(K)$ with the natural $I$-bundle structure. A surface of contact is a properly embedded compact surface $P$ in $N(B)$, transverse to the $I$-fibers, with $\partial P \subset$ $\partial_{v} N(B) \cup T$, such that the intersection of $\partial P$ with each component of the vertical surfaces $\partial_{v} N(B)$ is either empty or a single arc.

Let $p_{1}, p_{2}, n_{1}, n_{2}$ be the numbers of $a_{i}$ of type $P_{1}, P_{2}, N_{1}, N_{2}$ respectively. Let $r$ be the slope of $\partial R$ on $T$. Let

$$
J=\left\{r+\left(p_{1}-n_{1}\right) \frac{x}{x+1}+\left(p_{2}-n_{2}\right) x \mid x>0\right\}
$$

Then Roberts' theorem [Ro, Theorem 2.3] can be stated as

Theorem 5.1 (Roberts). If $B=\langle R, S\rangle$ constructed above is an essential branched surface in $E(K)$, and has no planar surface of contact, then $B$ extends to an essential branched surface $B_{\gamma}$ in $K(\gamma)$ for all slope $\gamma \in J$.

The construction of the extended branched surface is as follows. Let $T \times I$ be a small neighborhood of $T$ in $E(K)$ with $T=T \times 0$, such that $B \cap(T \times I)=\partial B \times I$. Add the digons $T \times 1-B$ to $B$, and branched so that the cusps on the two edges of each digon lie on different sides. The definition of $J$ guarantees that the train track $\partial B$ on $T$ can be split to produce a curve $C$ of slope $\gamma$ on $T$. Split $\partial B \times I$ accordingly and, after Dehn filling, cap off $C$ by a meridian disk in the Dehn filling solid torus, so that one obtains a branched surface $B_{\gamma}$ in $K(\gamma)$. It was shown in [Ro] that $B^{\prime}$ carries an essential lamination in $K(\gamma)$.

Denote by $E(B)$ the exterior of $B$ in $E(K)$, i.e $E(B)=E(K)-\operatorname{Int} N(B)$.

Corollary 5.2. For any $\gamma \in J$, the manifold $E(B)$ is homeomorphic to a component $W$ of the exterior of $B_{\gamma}$ in $K(\gamma)$, with horizontal surface of $B$ identified to the horizontal surface of $B_{\gamma}$ on $\partial W$. 

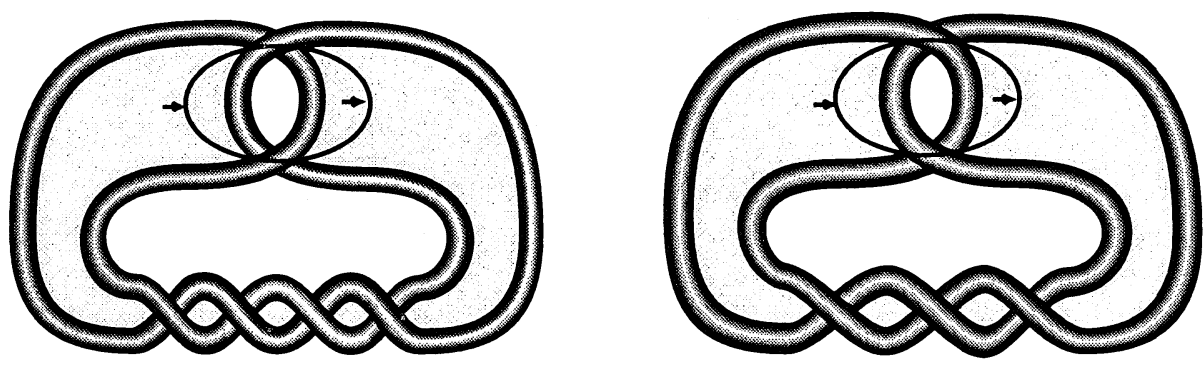

Figure 8.

Proof. Examine the above construction. After adding the digons of $T \times 1-B$ to $B$, the branched surface is topologically homeomorphic to $B \cup(T \times 1)$, which cuts off a region isotopic to $E(B)$. Clearly this region is not affected by the later changes, and its horizontal surface is the restriction of that on $E(B)$.

\section{Surgery on twist knots, and the final proof.}

As noticed earlier, any twist knot can be written as $K_{[2 n, \pm 2]}$ for some $n$, because if $b$ is odd then $K_{[b, 2]}=K_{[b-1,-2]}$. Since $K_{[2 n,-2]}$ is the mirror image of $K_{[-2 n, 2]}$, we need only consider knots of type $K_{[2 n, 2]}$. We can also assume that $n \neq 0,1$, otherwise the knot is a trivial knot or a trefoil knot.

Lemma 6.1. Let $K=K_{[2 n, 2]}$ be a twist knot with $n \neq 0,1$. Then $K(\gamma)$ is not a small Seifert fiber space for all $\gamma<-4$.

Proof. A knot $K=K_{[2 n, 2]}$ has two spanning surfaces as indicated in Figure 8 , where $2 n=4$. The first surface is a punctured Klein bottle, and the second one is a punctured torus.

Let $R$ be the punctured Klein bottle of Figure 8(1) in the knot exterior. Add a disk $S$ to $R$ such that the boundary of $S$ is the circle indicated in Figure 8(1). The boundary of $S$ consists of four arcs $a_{1} \cup b_{1} \cup a_{2} \cup b_{2}$, where $b_{i}$ lies on $R$, and $a_{i}$ on the torus $T=\partial N(K)$. The arrows at the $\operatorname{arcs} b_{i}$ indicate the side of the cusp. This determines the branched surface $B=\langle R, S\rangle$, and hence the type of $a_{i}$ on $T$. One of the $a_{i}$ (the one on top) is of type $P_{1}$, and the other one of type $N_{2}$. Using the notation in Section 5 , we have $p_{1}=n_{2}=1$, and $p_{2}=n_{1}=0$. By calculating the linking number between 


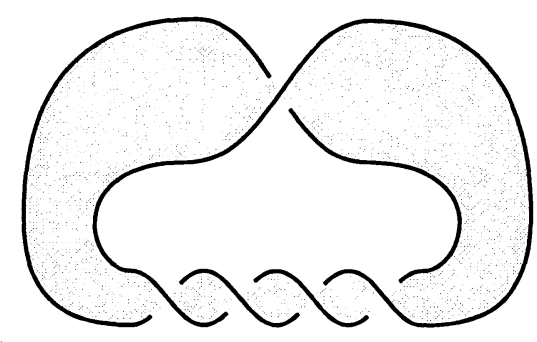

Figure 9.

$K$ and $\partial R$, one can see that $\partial R$ has slope $r=-4$ on $T$. Therefore,

$$
\begin{aligned}
J & =\left\{r+\left(p_{1}-n_{1}\right) \frac{x}{x+1}+\left(p_{2}-n_{2}\right) x \mid x>0\right\}=\left\{-4+\frac{x}{x+1}-x \mid x>0\right\} \\
& =\left\{-4-\frac{x^{2}}{x+1} \mid x>0\right\}=(-\infty,-4)
\end{aligned}
$$

We need to show that $B$ is an essential branched surface in $E(K)$. It is clear that $N(B)$ is topologically a solid torus. By examining the cusp on $\partial N(B)$, one can see that the exterior of $B$ is the same as that of the surface in Figure 9 , with cusp the boundary of the surface $F$. Note that $\partial F$ runs along the meridian of the solid torus $N(F) 2 n-1$ times. Hence $E(B)$, the exterior of $B$, is a solid torus with a single cusp running along the longitude $2 n-1$ times. Since $n \neq 0,1$, it is easy to see that $E(B)$ satisfies all conditions for $B$ to be essential, i.e, $E(B)$ is indecomposable, and the horizontal surface on $\partial E(B)$ is essential. One also needs to check that the branched surface $B$ satisfies all the intrinsic essentiality properties, i.e, it has no disk or half disk of contact, it contains no Reeb branched surfaces, and it fully carries a lamination. This is straightforward.

To apply Roberts' theorem, we also need to show that $B=\langle R, S\rangle$ has no planar surface of contact. Assume that $P$ is a compact surface embedded in $N(B)$, transverse to the $I$-fibers, with $\partial P \subset \partial_{v} N(B) \cup T$. Cutting along the two $\operatorname{arcs} b_{1}, b_{2}$ along which $S$ is glued to $R$, the branched surface $B$ becomes two surfaces $S$ and $R^{\prime}=R-b_{1} \cup b_{2}$. Let $u, v$ be the number of times that $P$ intersects an $I$-fiber of $S$ and $R^{\prime}$ respectively. Then the gluing at $b_{i}$ gives the equation $v=u+v+w$, where $w$ is the number of times $\partial P$ passes the cusp at $b_{i}$. So $u=w=0$, and $P$ lies in $N(R)$. Since $R$ is nonplanar, $P$ can not be planar.

It now follows from Theorem 5.1 that $B$ extends to an essential branched surface $B_{\gamma}$ for all slopes $\gamma<-4$. By Corollary 5.2, $E(B)$ can be considered 
as a component of the exterior of $B_{\gamma}$. Since $E(B)$ is a solid torus with a cusp running along the longitude $|2 n-1| \geq 3$ times, it is not an $I$-bundle. Therefore, by the result of Brittenham $[\mathrm{Br}], K(\gamma)$ is not a small Seifert fiber space for all $\gamma<-4$.

Lemma 6.2. Let $K=K_{[2 n, 2]}$ be a twist knot with $|n|>2$. Then $K(\gamma)$ is not a small Seifert fiber space for all $\gamma>0$.

Proof. The proof is very similar to that of Lemma 6.1, only instead of using the non orientable surface in Figure 8(1), we use the orientable surface $R$ in Figure 8(2). As a Seifert surface of $K$, the boundary of $R$ has slope 0 . Also, the $\operatorname{arcs} a_{1}, a_{2}$ are now of type $P_{2}$ and $N_{1}$, so $p_{1}=n_{2}=0, p_{2}=n_{1}=1$, and

$$
\begin{aligned}
J & =\left\{r+\left(p_{1}-n_{1}\right) \frac{x}{x+1}+\left(p_{2}-n_{2}\right) x \mid x>0\right\}=\left\{0-\frac{x}{x+1}+x \mid x>0\right\} \\
& =\left\{\frac{x^{2}}{x+1} \mid x>0\right\}=(0, \infty)
\end{aligned}
$$

The exterior of the branched surface $B=\langle R, S\rangle$ is the same as that of a band with $2 n$ twists. Since $|n|>2$, it is not an $I$-bundle. Therefore, one can use the argument in the proof of Lemma 6.1 to obtain the conclusion.

Proof of Theorem 1.1. Parts (1) and (2) are exactly Corollaries 3.4 and 4.2. For part (3), consider the knot $K_{[2 n, 2]}$. By Lemma 2.2 and Corollary 2.4, surgeries with $\gamma=0,4$ are the only toroidal ones, and $\gamma=-1,-2,-3$ are Seifert fibered. By Corollary 4.3, Lemmas 6.1 and 6.2, there are no other small Seifert fibered surgeries. Since no surgeries on $K_{[2 n, 2]}$ are reducible (Lemma 2.1), all surgeries with $\gamma \neq 0,-1,-2,-3,-4$ are non-exceptional. Since $K_{[2 n,-2]}$ is the mirror image of $K_{[-2 n, 2]}$, the result follows.

Part (4) follows from the well known result of Thurston about surgery on the Figure 8 knot [Th], which is stronger as the non-exceptional surgeries are shown to be hyperbolic. The result as stated can also be proved using the techniques in this paper: As toroidal and reducible surgeries are known, we only need to deal with small Seifert fibered surgeries. By $[\mathrm{Br}]$ all nonintegral surgeries on $K=K_{[-2,2]}$ are not small Seifert fibered. By Lemma $6.1 K(\gamma)$ are not small Seifert fibered for $\gamma<-4$. Since $K$ is amphicheiral, this is also true for $\gamma>4$. 
Remark 6.3. Boileau and Porti $[\mathrm{BP}]$ proved a version of Thurston's Orbifold Theorem, showing that the geometrization conjecture is true for manifolds which admit a finite group action whose fixed point set is a non-empty 1-manifold. Using this result one can show that non-exceptional surgeries on 2 -bridge knots are also hyperbolic. The proof is as follows. A $p / q 2$-bridge knot can be obtained by taking two arcs of slope $p / q$ on the "pillowcase", then joining the ends with two trivial arcs. From this picture it is easy to see that $K$ is a strongly invertible knot, i.e. there is an involution $\varphi$ of $S^{3}$ such that $\varphi(K)=K$, and the fixed point set of $\varphi$ is a circle $S$ intersecting $K$ at two points. The map $\varphi$ restricts to an involution of $E(K)=S^{3}-\operatorname{Int} N(K)$, which can be extended to an involution $\widehat{\varphi}$ of the surgered manifold. Since $\widehat{\varphi}$ has fixed point set a nonempty 1-manifold, the result follows from [BP].

\section{References.}

[BP] M. Boileau and J. Porti, Geometrization of 3-orbifolds of cyclic type, preprint.

[Br] M. Brittenham, Small Seifert-fibered spaces and Dehn surgery on 2-bridge knots, Topology 37 (1998), 665-672.

[De1] C. Delman, Essential laminations and Dehn surgery on 2-bridge knots, Topology and its Appl. 63 (1995), 201-221.

[De2] Constructing essential laminations which survive all Dehn surgeries, preprint.

[GO] D. Gabai and U. Oertel, Essential laminations in 3-manifolds, Annals of Math. 130 (1989), 41-73.

[G] C. Gordon, Dehn surgery on knots, Proceedings of the International Congress of Mathematicians, Kyoto 1990, 631-642.

[HT] A. Hatcher and W. Thurston, Incompressible surfaces in 2-bridge knot complements, Inv. Math. 79 (1985), 225-246.

[Lu] J. Luecke, Dehn surgery on knots in the 3-sphere, in Proceedings of the International Congress of Math. Vol. 1, 2 (Zurich, 1994), Birkhäuser, Basel $1995,585-594$.

[Mo] L. Moser, Elementary surgery along a torus knot, Pacific J. Math. 38 (1971), 737-745.

[Pr] J. Przytycki, Incompressibility of surfaces after Dehn surgery, Michigan Math. J. 30 (1983), 289-308. 
The classification of exceptional Dehn surgeries on 2-bridge knots 113

[Ro] R. Roberts, Constructing Taut Foliations, Comment. Math. Helv. 70 (1995), 516-545.

[R] D. Rolfsen, Knots and Links, Publish or Perish, Houston, 1990.

[Th] W. Thurston, The Geometry and Topology of 3-manifolds, Princeton University, 1978.

[Wu] Y-Q. Wu, Dehn surgery on arborescent links, Trans. Amer. Math. Soc., 351 (1999), 2275-2294.

RECEIVED OCTOBER 26, 1998.

UNIVERSITY OF NEBRASKA,

LINCOLN, NE 68588

AND

UNIVERSITY OF IOWA,

IOWA CITY, IA 52242

E-mail addresses: mbritten@math.unl.edu

wu@math.uiowa.edu 